\section{Zu genau hingesehen: Läuse am Auge}

Eine 36-jährige Frau litt seit etwa drei Wochen unter Juckreiz der Augenlider. Ihren Ehemann juckte es dagegen in der Schamregion. Bei der Lupenuntersuchung der Augenlider erkannte man einige wenige Läuse und Nissen an der Basis der Wimpern.

\begin{abstract}
A $\mathrm{m}$ rechten Auge bestand eine leichte konjunktivale Injektion, ansonsten war das Auge unauffällig. Im Bereich des Schamhaares der Frau fanden sich ebenfalls multiple Läuse und Nissen. Andere behaarte Regionen wie Augenbrauen, Achselhaar, Körperbehaarung oder Kopfhaut waren nicht befallen. Mikroskopisch erkannte man Phthirus pubis, vulgo Filzläuse, mit den dazu gehörigen Nissen. Ein Screening auf andere sexuell übertragbare Erkrankungen wie HIV, Syphilis, Hepatitis B und C erwies sich als unauffällig.

Die Läuse wurden mit einer Pinzette von den Wimpern entfernt und die Wimpern zweimal täglich mit Vaseline bestrichen. Die Haare der Schamregion wurden abrasiert und die Haut mit 10\%-iger
\end{abstract}

Schwefelsalbe einmal täglich über drei Tage hinweg behandelt. Die Patientin wurde angewiesen, alle Kleidungsstücke und sonstige Infektionsträger zu waschen. Ihr Gatte wurde in gleicher Weise behandelt. Zwei Wochen später hatte sich der Juckreiz bei beiden Personen zurückgebildet, Augenlider und Schamhaarbereich waren frei von Parasiten.

Fazit: Obwohl Phthirus pubis praktisch alle behaarten Stellen befallen kann, ist die Manifestation an den Augenlidern bei gesunden Erwachsenen doch extrem selten. In diesem Fall würde wohl die detaillierte Anamnese weiter helfen. Nachdem die Infektion bei den Erwachsenen in der Regel sexuell übertragen wird, empfiehlt sich ein Screening nach weiteren sexuell über-

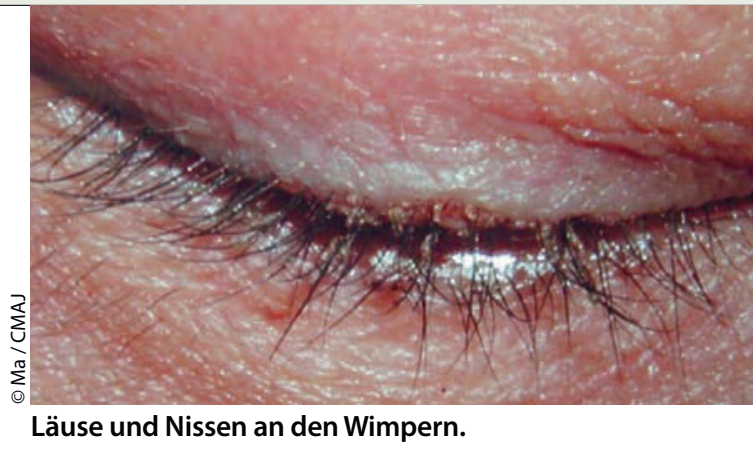

tragbaren Erkrankungen. Bei Kindern ist die Manifestation gerade an den Augenlidern aber besonders häufig. Vaseline ist sehr effektiv bei der Eradikation von Läusen, zerstört aber nicht die Eier. Sieben Tage nach Beginn der therapeutischen Maßnahme sollten die Haare erneut auf das Vorliegen von Filzläusen untersucht werden. Finden sich weitere Läuse, so ist ein anderes therapeutisches Regime anzuwenden, zum Beispiel Lindan, Permethrin, Malathion oder Phenothrin. Kleidung, Handtücher und Bettzeug sollten mit einer Temperatur von mindestens $55^{\circ} \mathrm{C}$ gewaschen werden.

H. S. FüeßI

Ma DL, Vano-Galvan S. Infestation of the eyelashes with Phthirus pubis. CMAJ 2010; 182: E187

\section{Wie richtige Fußpflege bei Senioren Stürze verhindern könnte}

\section{Krankheiten des Hallux können die Druckverteilung an der Fußsohle beim Gehen beeinträchtigen und theoretisch die gesamte Muskelleistung der unteren Extremitäten stören. Dermatologen der Universität Tokio wollten wissen, ob auch pathologische Veränderungen des Zehennagels die Funktionsfähigkeit der unteren Extremität beeinträchtigen können.}

$\mathrm{D}$ ie Studienpopulation (263 Erwachsene) setzte sich aus ambulanten Patienten der dermatologischen Abteilung mit Problemen an der großen Zehe und aus freiwilligen Probanden zusammen. Die Patienten litten unter Hallux valgus, eingewachsenen Zehennägeln oder Pachyonychie.

Mittels verschiedener Verfahren prüfte man das Steh- und Gehvermögen der Teilnehmer: Zur Beurteilung des Gleichgewichtsvermögens wurden Funktionstests (Einbein-Balancetest und Functional-reach-Test) herangezogen; mit einer speziellen Methode ermittelte man die Beinmuskelkraft. Es zeigte sich, dass das Vorliegen krankhafter Nagelverände- rungen die Muskelkraft und die Funktion der unteren Extremität beeinträchtigte; Druckschmerzhaftigkeit verstärkte die Fehlfunktion weiter.

55 der Patienten ließen ihre Füße daraufhin von medizinischen Fußpflegern versorgen. Das Programm umfasste warme Seifenbäder und konservative Maßnahmen wie „Packing“, das Einlegen von Watte unter den seitlichen distalen Nagelrand, Taping, also das Nach-außenZiehen des entzündeten Nagelwalls mittels eines Pflasterstreifens, sowie die Schienung des Nagels mittels eines Röhrchens. Verdickte Nagelplatten wurden abgeschliffen. Unmittelbar nach der Behandlung sowie vier Wochen später zeigten diese Patienten signifikant bessere Funktions-Scores. Die deutliche Schmerzreduktion durch die Behandlung hielt bis zu vier Wochen an.

Kontrollpersonen, die nicht podologisch behandelt wurden, konnten ihre Ergebnisse in den Funktionstests dagegen nicht verbessern.

Fazit: Angesichts der Tatsache, dass herabgesetzte muskuläre Leistung der unteren Extremitäten und eine verminderte Haltungskontrolle das Sturzrisiko älterer Menschen erhöhen, geht der Nutzen der Schmerzlinderung durch podologische Maßnahmen unter Umständen weit über das bisher angenommene Maß hinaus: Medizinische Fußpflege senkt nach Ansicht der Autoren im Endeffekt die Gefährdung der Patienten durch Stürze.

Waltraud Paukstadt

Imai A et al. Ingrown nails and pachyonychia of the great toes impair lower limb functions: improvement of limb dysfunction by medical foot care. Int J Dermatol 2011; 50: $215-20$ 